\title{
An adaptive threshold based CQI compression scheme for LTE cellular networks
}

\begin{abstract}
The frequency domain scheduling gain can be maximized when precise channel information is available at the eNodeB for the whole bandwidth. To compensate for the continuous variations of channel conditions in time and frequency domains, a huge undesirable amount of signaling overhead is required to report the channel quality indicator (CQI). On the contrary, partial channel state information detriments the downlink performances and does not guarantee the quality-of-service (QoS) when real-time multimedia services are applied. Thus, in this paper, the impact of CQI signaling overhead on the downlink performances is formulated. Then, an adaptive signal-to-interference-plus-noise ratio (SINR) threshold based CQI scheme is proposed by using multi-objective swarm intelligence to find the optimal feedback threshold. Based on an LTE system-level simulation, significant enhancements of $20 \%$ and $38 \%$ in throughput and packet loss ratio (PLR) respectively are obtained compared to a fixed threshold feedback with reasonable cost of feedback overhead. The proposed algorithm provides a high flexibility in responding to certain variations without complex modifications.
\end{abstract}

Keyword: Long term evolution (LTE); Channel quality indicator (CQI); Partial feedback 